\title{
Risk of Malignancies in Patients with Rheumatoid Arthritis Treated with Rituximab: Analyses of Global Postmarketing Safety Data and Long-Term Clinical Trial Data
}

Paul Emery · Daniel E. Furst · Petra Kirchner · Simone Melega •

Stuart Lacey · Patricia B. Lehane

Received: September 3, 2019 / Published online: November 21, 2019

(c) The Author(s) 2019

\section{ABSTRACT}

Introduction: Patients with rheumatoid arthritis (RA) are at an increased risk of developing malignancies, but it is unclear whether this increased risk is the result of disease pathobiology or immunosuppressant treatments for RA. This analysis evaluated the potential risk of malignancy in patients with RA treated with rituximab $\left(\right.$ MabThera ${ }^{\circledR} /$ Rituxan $^{\circledR}$ ) a CD20+

Enhanced Digital Features To view enhanced digital features for this article go to https://doi.org/10.6084/ m9.figshare.10078130.

P. Emery

NIHR Leeds Musculoskeletal Biomedical Research Unit, Leeds Teaching Hospitals NHS Trust, Leeds Institute of Rheumatic and Musculoskeletal

Medicine, Leeds University, Leeds, UK

D. E. Furst

University of California, Los Angeles, Los Angeles, CA, USA

D. E. Furst

University of Washington, Seattle, WA, USA

D. E. Furst

University of Florence, Florence, Italy

P. Kirchner · S. Melega

F. Hoffmann-La Roche Ltd, Basel, Switzerland

S. Lacey · P. B. Lehane $(\bowtie)$

Roche Products Ltd, Welwyn Garden City, UK e-mail: patricia.lehane@roche.com
B-cell depleting agent manufactured by $\mathrm{F}$. Hoffmann-La Roche Ltd.

Methods: Malignancy rates were obtained from the rituximab global company safety database for adverse event reporting and from the rituximab global clinical trial program for RA consisting of eight randomized clinical trials, two long-term open-label extensions, and one openlabel prospective study. Global company safety database searches were performed using the standard Medical Dictionary for Regulatory Activities (MedDRA) queries "Malignant tumors wide" and "Skin malignant tumors wide" up to April 30, 2017. Age- and sex-specific comparator values from the general population were obtained from the US National Cancer Institute Surveillance, Epidemiology, and End Results (SEER) database.

Results: For the 409,706 patients with RA in the rituximab global company safety database since first market approval in 2006, 1739 cumulative malignant events were reported, with an overall malignancy reporting rate of approximately 4.2 events per 1000 patients. No evidence of increased risk of malignancy, of any organ-specific type, was found following rituximab treatment. The rate of malignancies from rituximab-treated patients in RA clinical trials was 7.4 per 1000 patient-years. This is within the expected range, with no evidence for increased risk over time or with additional rituximab courses. 
Conclusions: Analyses of the global postmarketing safety database and long-term clinical trial data showed no evidence of an increased risk of malignancy of any type following rituximab treatment in patients with RA.

Funding: F. Hoffmann-La Roche Ltd.

Keywords: Database; Malignancy; Real-world; Rheumatoid arthritis; Rituximab; Safety

\section{Key Summary Points}

\section{Why carry out this study?}

Patients with rheumatoid arthritis (RA) are at an increased risk of developing malignancies, but it is unclear whether this increased risk is the result of the disease pathobiology or treatment for their arthritis.

This analysis evaluated the potential risk of malignancy in patients with RA treated with rituximab using spontaneous postmarketing data and available clinical trial data.

\section{What was learned from the study?}

No evidence of an increased risk of malignancy of any organ-specific type following rituximab treatment was found in analysis of the rituximab global postmarketing safety database over 11 years since first market approval in RA.

The rate of malignancies from rituximabtreated patients in RA clinical trials was within the expected range, with no evidence for increased risk over time or with additional rituximab courses.

The overall malignancy reporting rate and malignancy types were consistent with those expected in patients with RA.

\section{INTRODUCTION}

The anti-CD20 monoclonal antibody rituximab (MabThera $^{\circledR} /$ Rituxan $^{\circledR}$, manufactured by F.
Hoffmann-La Roche Ltd) targets and depletes $\mathrm{CD} 20+\mathrm{B}$ cells and is a biologic immunosuppressive therapy indicated for use in rheumatoid arthritis (RA) since 2006 [1, 2]. Rituximab in combination with methotrexate (MTX) improves disease symptoms in patients with moderate-to-severe, active RA who responded inadequately to $\geq 1$ antitumor necrosis factor agent (aTNF). Rituximab also reduces the rate of progression of joint damage as measured by X-ray and improves physical function [3-10].

Long-term follow-up data from rituximab trials have demonstrated a well-tolerated safety profile in patients with RA (up to 11 years' follow-up) [11] as well as in patients with antineutrophil cytoplasmic antibody (ANCA)associated vasculitis (up to 5 years' follow-up), for which rituximab is also indicated $[12,13]$. Although these data are encouraging, it is important to continue monitoring the longterm safety of rituximab in patients with autoimmune diseases to identify potential cumulative risks associated with prolonged and repeated peripheral blood B-cell depletion, such as immunosuppression-associated malignancy.

Patients with RA have a slightly higher overall malignancy risk than the general population due to an elevated risk of specific malignancies, including lymphoma, lung cancer, and nonmelanoma skin cancer (NMSC) [14-16]. It is unclear whether this increased malignancy risk is a consequence of the underlying inflammatory activity associated with the disease itself or the immunosuppressive therapies used to treat RA. Several studies in patients with RA have reported no additional risk of overall malignancy with disease-modifying antirheumatic drugs (DMARDs) and biologics, including aTNFs [17-20], Janus kinase inhibitors [21], tocilizumab [22, 23], and rituximab [17, 20]. However, there are conflicting findings concerning the risk of NMSC with aTNF therapy [18-20, 24-26].

A recent analysis of data from patients with ANCA-associated vasculitis treated with rituximab found no increase in malignancy risk compared with that in the general population [27]. Similarly, there was no increased risk of malignancy in patients with RA treated with rituximab in long-term clinical trials over 11 years compared with that observed in the 
general population or in published data in adults with RA [11]. The aim of our analysis is to further evaluate and characterize the potential risk of malignancy in patients with RA treated with rituximab using spontaneous postmarketing data and available clinical trial data.

\section{METHODS}

\section{Analysis of the Rituximab Global Company Safety Database in RA}

The global company safety database includes all serious and nonserious cases from spontaneous sources where rituximab is considered "suspect" (irrespective of reporter and company causality assessment), in addition to the serious adverse events and designated nonserious adverse events reported from global clinical trials. To provide cumulative malignant events for patients receiving rituximab for RA, the global company safety database was searched up to June 17, 2014, using the standard Medical Dictionary for Regulatory Activities (MedDRA; Version 17.0), with a subsequent search covering the period of June 18, 2014, to April 30, 2017 (MedDRA Version 20.0). The search used the standardized MedDRA queries (SMQs) "Malignant tumors wide" and "Skin malignant tumors wide." Cumulative data for the five most frequently reported high-level group terms are presented, including the most frequently reported MedDRA preferred terms and patient characteristics (sex, age, and history of prior malignancy). Incidence rates of malignant events could not be estimated, as it was not possible to accurately calculate overall patient exposure from the global safety database.

Based on the potentially increased risk of skin cancer or NMSC in patients with RA treated with biologics [26], additional searches and analyses were conducted for patients with skin malignancies. The SMQ "Skin malignant tumors wide" was used to broadly cover all skin malignant tumor preferred terms, including preferred terms of interest for further evaluation of NMSC (basal cell carcinoma, squamous cell carcinoma of skin, Bowen disease) and skin cancer. Cases with reported preferred terms of NMSC and skin cancer were analyzed further at the case level.

Analyses conducted to investigate any causative link between NMSC and rituximab took into account the following factors: latency of onset from first dose of rituximab and from most recent dose of rituximab, rituximab therapy duration, and cumulative dose of rituximab before the onset of the NMSC event; latency of other immunosuppressants (first/last dose); ethnicity; concomitant medications as risk factors; other known risk factors; history of malignancy/concurrent malignancy; and diagnosis confirmed by a biopsy.

Analysis of the rituximab global company safety database for RA was an observational study that used only de-identified patient records and did not involve the collection, use, or transmittal of individually identifiable data; therefore, institutional review board approval to conduct this analysis was not necessary. The analysis of the RA clinical trial database was a pooled observed case analysis of safety data from patients who participated in the RA clinical trial program. Study designs and results of the clinical trials have been previously published and the study protocols for each clinical trial were approved by an ethics committee or institutional review board at each participating center before the start of each study. All patients provided written informed consent in accordance with the Declaration of Helsinki.

\section{Analysis of the RA Global Clinical Trial Database}

The RA clinical trial database included patients with moderate-to-severe active RA treated with rituximab plus MTX enrolled in a global clinical trial program, which included MTX-naive patients as well as patients with an inadequate response to prior DMARDs including aTNFs. It comprised eight randomized clinical trials, two long-term open-label extensions, and one openlabel prospective study $[3-10,28,29]$ as of September 2012 (final clinical cut-off date). Eligibility criteria, study designs, and treatment regimens for these trials have been previously published [3-10, 28, 29]. The rituximab all- 
exposure population consisted of all patients exposed to at least one or part of one rituximab infusion, regardless of dose. The clinical database of the all-exposure RA population was searched for malignancy events using the SMQ (MedDRA Version 16.0) "Malignant tumors wide."

The analysis of the RA clinical trial database was a pooled observed case analysis of safety data from patients who participated in the RA clinical trial program. Study designs and results of the clinical trials have been previously published and the study protocols for each clinical trial were approved by an ethics committee or institutional review board at each participating center before the start of each study. All patients provided written informed consent in accordance with the Declaration of Helsinki.

\section{Statistical Analyses}

Malignancy rates are reported as events per 1000 patient-years (PY). Standardized incidence ratios (SIRs) were calculated as the ratio of number of observed malignancies to expected malignancies based on background rates within general population samples. Age- and sexspecific reference values of non-NMSC malignancy rates in patients with RA and in the general US population were obtained from previously published reports [11] and from the US National Cancer Institute Surveillance, Epidemiology, and End Results (SEER) database [30], respectively. Where available, 95\% confidence intervals (CIs) are reported.

\section{RESULTS}

\section{Rituximab Global Company Safety Database}

As of April 30, 2017, 409,706 patients with RA had been exposed to rituximab since first market approval in 2006. A total of 1739 cumulative malignant events were reported, corresponding to an overall malignancy reporting rate of approximately 4.2 events per 1000 patients. Based on the MedDRA query search, the high- level group terms with the highest number of events (malignant and unspecified) were skin neoplasms with 303 events (from 273 cases, of which 57 patients had a history of prior malignancy), followed by breast neoplasms (including nipple) with 236 events (from 222 cases, of which 30 patients had a history of prior malignancy), and respiratory and mediastinal neoplasms with 231 events (from 223 cases, of which 53 had a history of prior malignancy) (Table 1). The most common events reported by MedDRA preferred term were breast cancer with 157 events followed by basal cell carcinoma with 124 events. The five most common highlevel group terms are summarized in Table 1, along with the most frequently reported events, demographic characteristics, and malignancy history of the respective patients.

None of the identified NMSC cases were considered to have a possible causal association with rituximab based on analysis by the marketing authorization holder. No consistent timing of occurrence of an NMSC event relative to the timing or duration of prior treatment with rituximab was identified. Risk factors such as underlying disease, use of immunosuppressants, and medical history of malignant or premalignant skin conditions were identified in all NMSC cases.

\section{RA Global Clinical Trial Database}

According to the final long-term safety report of the RA global clinical trial program [11], 3595 patients were included in the RA all-exposure rituximab population ( $80 \%$ female; mean age, 51.8 years) and received a mean of four courses (range, 1-20) of rituximab over 11 years $(14,816$ PY). There was no evidence of an increased risk of malignancy of any type over time or by increased number of rituximab courses (patients with a history of prior malignancy were excluded from study entry).

As previously reported [11], the rate of overall confirmed malignancies (excluding NMSC and nonmalignant events) (109 total events, 7.4 per 1000 PY [95\% CI, 6.0-8.8]) was comparable with or lower than rates observed in the general RA population (11.7 per 1000 PY 


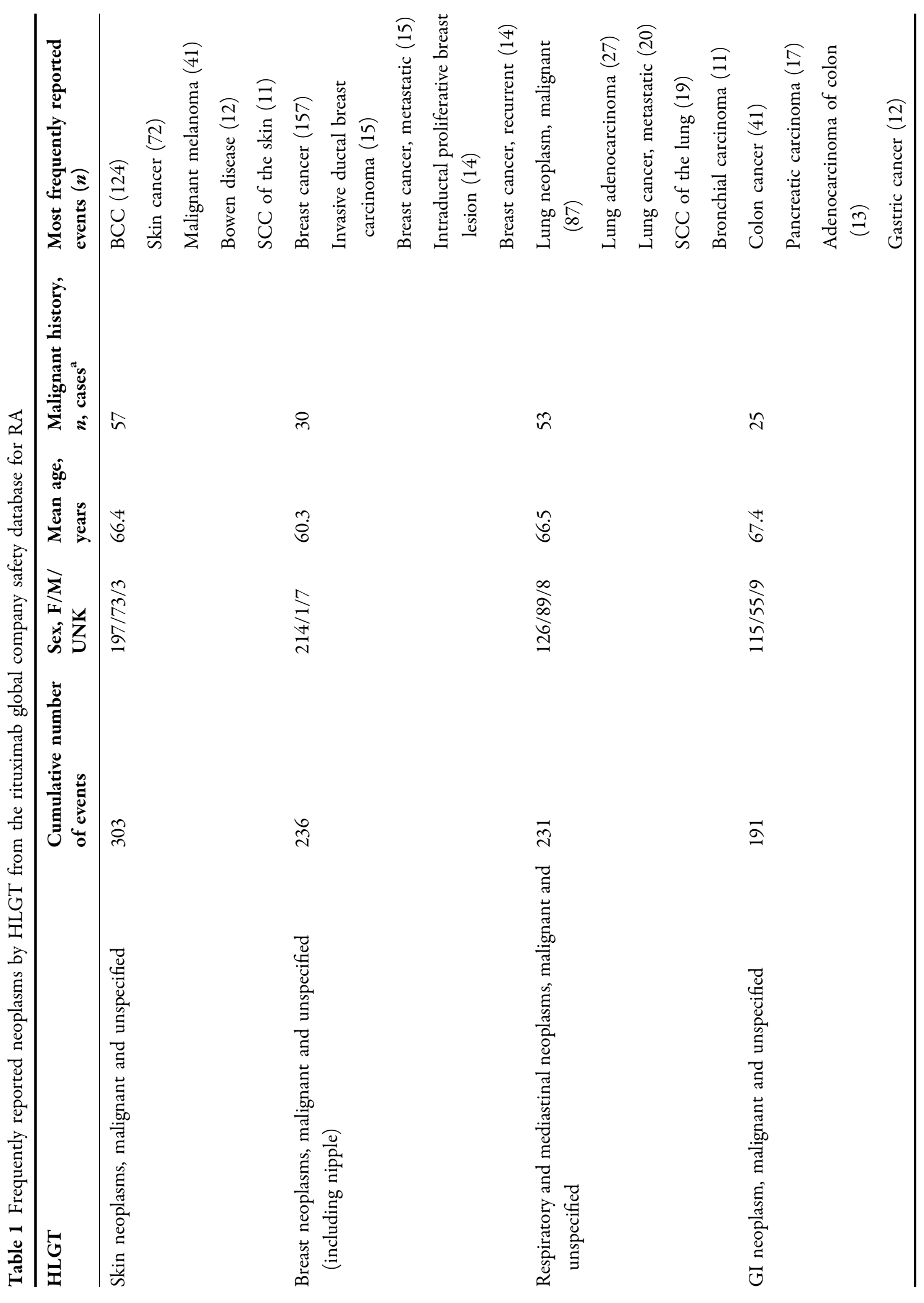




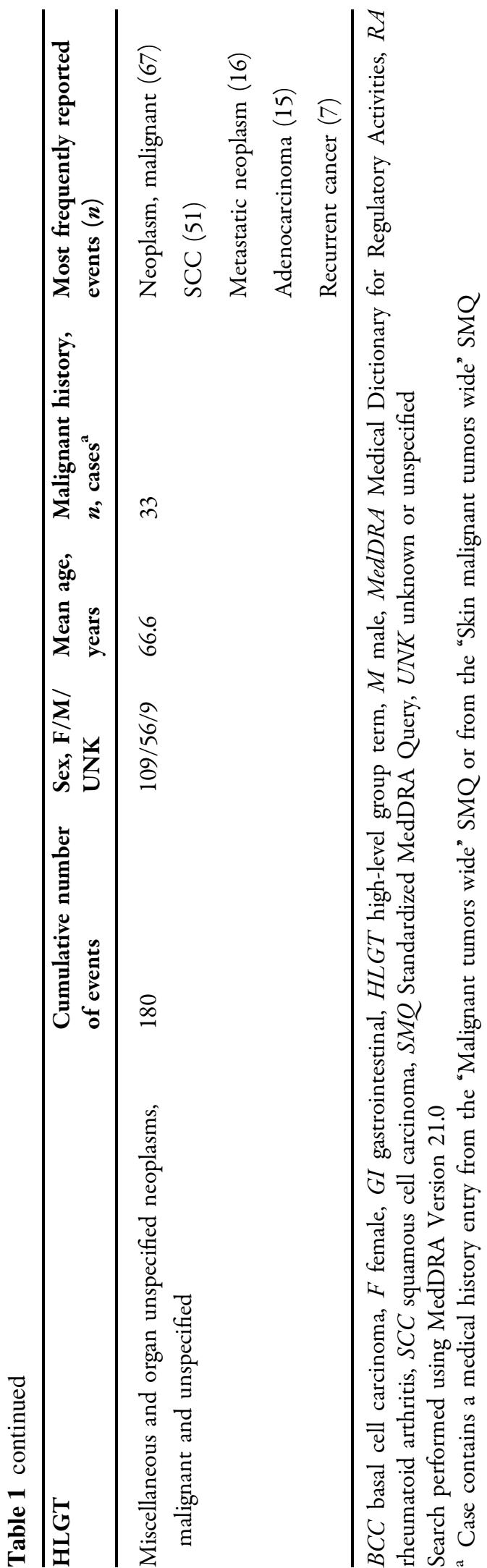

and 13.0 per 1000 PY [95\% CI, 11.9-14.1]) $[26,31]$. Breast cancer was the most frequently reported malignancy (16 total events, 1.4 per 1000 PY [95\% CI, 0.8-2.2], in female patients only), with a rate that was comparable with or lower than that reported in the general adult RA population (1.3 per $1000 \mathrm{PY}$ and 2.1 per $1000 \mathrm{PY}$ [95\% CI, 1.7-2.6]) [26, 31]. The rates of overall confirmed malignancies and breast cancer did not increase over time (Table 2). As previously reported [11], age- and sex-matched SIRs for non-NMSC malignancies (1.07 [95\% CI, 0.88-1.29]) were comparable with published data in adults with RA $(1.05$ [95\% CI, 1.01-1.09]) [16] and with data obtained from the SEER database (1.1 [95\% CI, 0.9-1.3]) [30] of the general US population. Similarly, the SIR for breast cancer (0.63 [95\% CI, 0.36-1.03]) was comparable with that from published data in adults with RA (0.84 [95\% CI, 0.79-0.90]) [16].

Among 68 NMSC events reported (rate 4.6 per 1000 PY) [11], basal cell carcinoma was most frequent (46 events), followed by squamous cell carcinoma (21 events).

There were $\leq 6$ events each of other organspecific malignancies, including lymphoma, leukemia, kidney, liver, ovarian, and prostate cancers, falling within or below the expected rate ranges for this population [26] (data not shown).

\section{DISCUSSION}

This study represents the most comprehensive analysis of long-term malignancy reporting rates in patients treated with rituximab for RA, drawing on all spontaneously reported safety events since 2006 and RA clinical trials covering a period up to 11 years of follow-up. Analysis of organ-specific malignancies of interest in patients with RA found no increased risk with rituximab treatment. Breast cancer was the most frequently reported malignant event in the rituximab global company safety database and the most frequently reported solid tumor observed in the clinical trial program. However, reported rates did not differ from those of the general population of adults with RA [32]. Skin neoplasms were the most frequent high-level 
Table 2 Rates of all malignancies and breast cancer over time from RA clinical trials (all-exposure rituximab population)

\begin{tabular}{llll}
\hline $\begin{array}{l}\text { Year } \\
\text { of data } \\
\text { cut-off }\end{array}$ & $\begin{array}{l}\text { Total number } \\
\text { of patients } \\
\text { (exposure in } \\
\text { PY) }\end{array}$ & $\begin{array}{l}\text { Rate of overall } \\
\text { malignancy } \\
\text { per 1000 PY }\end{array}$ & $\begin{array}{l}\text { Rate of } \\
\text { breast } \\
\text { cancer per } \\
\mathbf{1 0 0 0}_{\mathbf{P Y}}\end{array}$ \\
\hline 2007 & $2578(4006)$ & 8.2 & 2.2 \\
2008 & $3095(7198)$ & 8.3 & 1.4 \\
2009 & $3189(9342)$ & 8.2 & 1.4 \\
2010 & $3194(11,962)$ & 6.9 & 1.2 \\
2011 & $3595(14,008)$ & 7.2 & 1.1 \\
2012 & $3595(14,816)$ & 7.4 & 1.4 \\
\hline
\end{tabular}

$P Y$ patient-years, $R A$ rheumatoid arthritis, NMSC nonmelanoma skin cancer

a Serious adverse events as reported by the investigator; excludes NMSC and nonmalignant events

${ }^{b}$ Female patients only

group terms in the rituximab global company safety database, of which the majority were NMSC, a type of malignancy previously suggested to be increased by exposure to biologics $[19,24,26]$. The incidence rate of NMSC in the rituximab clinical trial program (4.6 per 1000 PY) [11] was lower than those reported in patients with RA who received aTNFs (18.9 per 1000 PY), nonbiologic DMARDs (12.7 per 1000 PY) [33], as well as other therapies (18.1 per 1000 PY) [34]. In addition, a detailed review of NMSC cases in the safety database did not reveal any specific pattern and was consistent with epidemiology and literature reports in nononcology indications, indicating no additional risk of NMSC related to rituximab [16, 30].

The extended analysis of long-term followup data from the rituximab clinical trial program in RA [11] showed no trend toward increased overall risk of malignancies with rituximab over time. These robust data pooled from randomized controlled trials provide information from a frequently assessed patient population in which significant comorbidities such as prior malignancy were excluded, reducing confounding factors. Importantly, the lack of increased malignancy risk with rituximab in patients with RA was supported by analysis of the global safety database, with less stringent inclusion criteria. Findings from the British Society of Rheumatology Biologics Register (BSRBR) and the Swedish Rheumatology Quality/Anti-Rheumatic Therapy in Sweden (SRQ/ARTIS) registries [20, 35] and recent data from the SUNSTONE registry [36] further support the conclusion that malignancy risk in patients with RA is not increased by treatment with rituximab compared with nonbiologic treatment.

A strength of this analysis is the presentation of safety data from both the global company safety database and the RA clinical trials database. Although an incidence rate could be calculated for the clinical trials database, patients with significant comorbidities are excluded from clinical trials. The spontaneously reported data from the global safety database, on the other hand, included patients with any comorbidities and pre-existing conditions but could not yield an incidence rate due to the inability to accurately calculate overall patient exposure. In addition, because safety data for rituximab are widely known, there may be a channeling bias related to treatment, with patients at higher risk of malignancy or recurrence being treated with rituximab $[6,8,11,17]$. This may result in an increased rate of malignancy in observational databases. Thus, the sum of this report gives the most complete picture of malignancies in patients with RA exposed to rituximab with the data currently available.

A major confounding factor in the analysis of malignancy in patients with RA following rituximab treatment is that many patients with RA receive rituximab therapy after treatment with other DMARDs or biologics for RA [37, 38]. Many patients had received prior DMARDs, including aTNF therapy, prior to enrollment and, therefore, had a cumulative exposure to biologic treatment longer than the stated follow-up periods. Indeed, aTNF treatment may increase the risk of NMSC $[18,19,24,26]$. An additional limitation of the analysis is that patients stopping rituximab therapy are more likely to have been lost to follow-up than those staying on therapy, which may have led to an 
exclusion of certain patient sub-populations within this analysis. It is also possible that some cases of malignancy among patients with RA receiving rituximab may not have been reported to the rituximab global company safety database and therefore were not included in this analysis. Finally, the SEER database that was used for comparison of age- and sex-matched SIRs contained data from the US general population whereas clinical trial data were obtained from patients with RA in multiple global countries.

\section{CONCLUSIONS}

This safety analysis of the global company postmarketing safety database and long-term clinical trial data identified no additional increased risk of malignancy of any type with long-term use of rituximab treatment among patients with RA. Malignancy reporting rates from patients in RA clinical trials remained stable over time, and malignancy types were consistent with those expected in patients with RA.

\section{ACKNOWLEDGEMENTS}

The authors thank the patients, investigators, and investigative staff who contributed to the rituximab global company safety database and who participated in the rituximab global RA clinical trial program.

Funding. This work was funded by F. Hoffmann-La Roche Ltd. All authors had full access to all of the data in this study and take complete responsibility for the integrity of the data and accuracy of the data analysis. The Rapid Service Fee were funded by F. Hoffmann-La Roche Ltd.

Medical Writing, Editorial, and Other Assistance. Support for third-party writing assistance, furnished by Susan Parker of Fishawack Indicia Ltd and Nicola Gillespie, DVM, of Health Interactions, Inc, was provided by $\mathrm{F}$. Hoffmann-La Roche Ltd.
Authorship. All named authors meet the International Committee of Medical Journal Editors (ICMJE) criteria for authorship for this article, take responsibility for the integrity of the work as a whole, and have given their approval for this version to be published.

Authorship Contributions. All authors were involved in drafting the manuscript, reviewing, and revising it critically for important intellectual content. Paul Emery, Daniel Furst, Patricia Lehane, Petra Kirchner, and Simone Melega contributed to the conception and study design, acquisition, analysis, and interpretation of data. Stuart Lacey contributed to the study design, analysis, and interpretation of data. All authors read and approved the final version.

Disclosures. Paul Emery has undertaken clinical trials and provided expert advice to AbbVie, BMS, Gilead, Lilly, MSD, Novartis, Pfizer, Roche, Samsung, Sandoz, and UCB. Daniel Furst has received grant/research support from BMS, Pfizer, and Roche/Genentech, and has been a consultant for AbbVie, Novartis, Pfizer, and Roche/Genentech. Petra Kirchner is an employee of F. Hoffmann-La Roche Ltd. Simone Melega is an employee of F. Hoffmann-La Roche Ltd. Stuart Lacey is an employee of Roche Products Ltd. Patricia Lehane is an employee of Roche Products Ltd.

Compliance with Ethics Guidelines. Analysis of the rituximab global company safety database for RA was an observational study that used only de-identified patient records and did not involve the collection, use, or transmittal of individually identifiable data; therefore, institutional review board approval to conduct this analysis was not necessary. The analysis of the RA clinical trial database was a pooled observed case analysis of safety data from patients who participated in the RA clinical trial program. Study designs and results of the clinical trials have been previously published and the study protocols for each clinical trial were approved by an ethics committee or institutional review board at each participating center before the start of each study. All patients provided written 
informed consent in accordance with the Declaration of Helsinki.

Data Availability. Access to individual patient-level data from the datasets used and/or analyzed during the current study may be requested by qualified researchers through the clinical study data request platform (www. clinicalstudydatarequest.com). Further details on Roche's criteria for eligible studies are available here: (https://clinicalstudydatarequest. com/Study-Sponsors/Study-Sponsors-Roche.aspx). For further details on Roche's Global Policy on the Sharing of Clinical Information and how to request access to related clinical study documents, see here: (https://www.roche.com/research_and_ development/who_we_are_how_we_work/clin ical_trials/our_commitment_to_data_sharing.htm).

Open Access. This article is distributed under the terms of the Creative Commons Attribution-NonCommercial 4.0 International License (http://creativecommons.org/licenses/ by-nc/4.0/), which permits any noncommercial use, distribution, and reproduction in any medium, provided you give appropriate credit to the original author(s) and the source, provide a link to the Creative Commons license, and indicate if changes were made.

\section{REFERENCES}

1. Rituxan (rituximab) injection, for intravenous use: US prescribing information. South San Francisco, CA: Genentech, Inc. 2018.

2. MabThera. Summary of product information. Grenzach-Wyhlen, Germany: Roche Registration GmbH. 2019.

3. Cohen SB, Emery P, Greenwald MW, Dougados M, Furie RA, Genovese MC, et al. Rituximab for rheumatoid arthritis refractory to anti-tumor necrosis factor therapy: results of a multicenter, randomized, double-blind, placebo-controlled, phase III trial evaluating primary efficacy and safety at twenty-four weeks. Arthritis Rheumatol. 2006;54(9):2793-806.

4. Edwards JCW, Szczepański L, Szechiński J, Filipowicz-Sosnowska A, Emery P, Close DR, et al. Efficacy of B-cell-targeted therapy with rituximab in patients with rheumatoid arthritis. N Engl J Med. 2004;350(25):2572-81.

5. Emery P, Fleischmann R, Filipowicz-Sosnowska A, Schechtman J, Szczepanski L, Kavanaugh A, et al. The efficacy and safety of rituximab in patients with active rheumatoid arthritis despite methotrexate treatment: results of a phase IIB randomized, double-blind, placebo-controlled, dose-ranging trial. Arthritis Rheumatol. 2006;54(5):1390-400.

6. Emery P, Deodhar A, Rigby WF, Isaacs JD, Combe B, Racewicz AJ, et al. Efficacy and safety of different doses and retreatment of rituximab: a randomised, placebo-controlled trial in patients who are biological naive with active rheumatoid arthritis and an inadequate response to methotrexate (Study Evaluating Rituximab's Efficacy in MTX iNadequate rEsponders [SERENE]). Ann Rheum Dis. 2010;69(9): 1629-35.

7. Keystone E, Fleischmann R, Emery P, Furst DE, van Vollenhoven R, Bathon J, et al. Safety and efficacy of additional courses of rituximab in patients with active rheumatoid arthritis: an open-label extension analysis. Arthritis Rheum. 2007;56(12): 3896-908.

8. Mease PJ, Cohen S, Gaylis NB, Chubick A, Kaell AT, Greenwald $\mathrm{M}$, et al. Efficacy and safety of retreatment in patients with rheumatoid arthritis with previous inadequate response to tumor necrosis factor inhibitors: results from the SUNRISE trial. J Rheumatol. 2010;37(5):917-27.

9. Rubbert-Roth A, Tak PP, Zerbini C, Tremblay JL, Carreno L, Armstrong G, et al. Efficacy and safety of various repeat treatment dosing regimens of rituximab in patients with active rheumatoid arthritis: results of a phase III randomized study (MIRROR). Rheumatology (Oxford). 2010;49(9):1683-93.

10. Tak PP, Rigby WF, Rubbert-Roth A, Peterfy CG, van Vollenhoven RF, Stohl W, et al. Inhibition of joint damage and improved clinical outcomes with rituximab plus methotrexate in early active rheumatoid arthritis: the IMAGE trial. Ann Rheum Dis. $2011 ; 70(1): 39-46$.

11. van Vollenhoven RF, Fleischmann RM, Furst DE, Lacey S, Lehane PB. Long-term safety of rituximab: final report of the rheumatoid arthritis global clinical trial program over 11 years. J Rheumatol. 2015;42(10):1761-6.

12. Alberici F, Smith RM, Jones RB, Roberts DM, Willcocks LC, Chaudhry A, et al. Long-term follow-up of patients who received repeat-dose rituximab as maintenance therapy for ANCA-associated vasculitis. Rheumatology (Oxford). 2015;54(7):1153-60. 
13. Terrier B, Pagnoux C, Perrodeau E, Karras A, Khouatra C, Aumaître $\mathrm{O}$ et al. Rituximab versus azathioprine to maintain remission of ANCA-associated vasculitides (MAINRITSAN): follow-up at 60 months [abstract]. Arthritis Rheumatol. 2016;68 (suppl 10).

14. Askling J, Fored CM, Brandt L, Baecklund E, Bertilsson L, Feltelius N, et al. Risks of solid cancers in patients with rheumatoid arthritis and after treatment with tumour necrosis factor antagonists. Ann Rheum Dis. 2005;64(10):1421-6.

15. Simon TA, Thompson A, Gandhi KK, Hochberg MC, Suissa $S$. Incidence of malignancy in adult patients with rheumatoid arthritis: a meta-analysis. Arthritis Res Ther. 2015;17(1):212.

16. Smitten AL, Simon TA, Hochberg MC, Suissa S. A meta-analysis of the incidence of malignancy in adult patients with rheumatoid arthritis. Arthritis Res Ther. 2008;10(2):R45.

17. Aaltonen KJ, Joensuu JT, Virkki L, Sokka T, Aronen $\mathrm{P}$, Relas $\mathrm{H}$, et al. Rates of serious infections and malignancies among patients with rheumatoid arthritis receiving either tumor necrosis factor inhibitor or rituximab therapy. J Rheumatol. 2015;42(3):372-8.

18. Chen Y, Friedman M, Liu G, Deodhar A, Chu CQ. Do tumor necrosis factor inhibitors increase cancer risk in patients with chronic immune-mediated inflammatory disorders? Cytokine. 2018;101: 78-88.

19. Lopez-Olivo MA, Tayar JH, Martinez-Lopez JA, Pollono EN, Cueto JP, Gonzales-Crespo MR, et al. Risk of malignancies in patients with rheumatoid arthritis treated with biologic therapy: a metaanalysis. JAMA. 2012;308(9):898-908.

20. Wadström H, Frisell T, Askling J. Malignant neoplasms in patients with rheumatoid arthritis treated with tumor necrosis factor inhibitors, tocilizumab, abatacept, or rituximab in clinical practice: a nationwide cohort study from Sweden. JAMA Intern Med. 2017;177(11):1605-12.

21. Curtis JR, Lee EB, Kaplan IV, Kwok K, Geier J, Benda B, et al. Tofacitinib, an oral Janus kinase inhibitor: analysis of malignancies across the rheumatoid arthritis clinical development programme. Ann Rheum Dis. 2016;75(5):831-41.

22. Kim SC, Pawar A, Desai RJ, Solomon DH, Gale S, Bao M, et al. Risk of malignancy associated with use of tocilizumab versus other biologics in patients with rheumatoid arthritis: a multi-database cohort study. Semin Arthritis Rheum. 2019;49(2):222-8.
23. Rubbert-Roth A, Sebba A, Brockwell L, Kelman A, Porter-Brown B, Pulley J, et al. Malignancy rates in patients with rheumatoid arthritis treated with tocilizumab. RMD Open. 2016;2(1):e000213.

24. Mariette X, Matucci-Cerinic M, Pavelka K, Taylor P, van Vollenhoven R, Heatley R, et al. Malignancies associated with tumour necrosis factor inhibitors in registries and prospective observational studies: a systematic review and meta-analysis. Ann Rheum Dis. 2011;70(11):1895-904.

25. Ramiro S, Sepriano A, Chatzidionysiou K, Nam JL, Smolen JS, van der Heijde D, et al. Safety of synthetic and biological DMARDs: a systematic literature review informing the 2016 update of the EULAR recommendations for management of rheumatoid arthritis. Ann Rheum Dis. 2017;76(6): 1101-36.

26. Wolfe F, Michaud K. Biologic treatment of rheumatoid arthritis and the risk of malignancy: analyses from a large US observational study. Arthritis Rheum. 2007;56(9):2886-95.

27. van Daalen EE, Rizzo R, Kronbichler A, Wolterbeek $\mathrm{R}$, Bruijn JA, Jayne DR, et al. Effect of rituximab on malignancy risk in patients with ANCA-associated vasculitis. Ann Rheum Dis. 2017;76(6):1064-9.

28. Bingham CO 3rd, Looney RJ, Deodhar A, Halsey N, Greenwald $\mathrm{M}$, Codding $\mathrm{C}$, et al. Immunization responses in rheumatoid arthritis patients treated with rituximab: results from a controlled clinical trial. Arthritis Rheum. 2010;62(1):64-74.

29. van Vollenhoven RF, Emery P, Bingham CO 3rd, Keystone EC, Fleischmann RM, Furst DE, et al. Long-term safety of rituximab in rheumatoid arthritis: 9.5-year follow-up of the global clinical trial programme with a focus on adverse events of interest in RA patients. Ann Rheum Dis. 2013;72(9): 1496-502.

30. National Cancer Institute. Surveillance, Epidemiology, and End Results. [Internet. Accessed June 30, 2015.]. seer.cancer.gov.

31. Mellemkjaer L, Linet MS, Gridley G, Frisch M, Moller H, Olsen JH. Rheumatoid arthritis and cancer risk. Eur J Cancer. 1996;32A(10):1753-7.

32. Stone JH, Merkel PA, Spiera R, Seo P, Langford CA, Hoffman GS, et al. Rituximab versus cyclophosphamide for ANCA-associated vasculitis. N Engl J Med. 2010;363(3):221-32.

33. Amari W, Zeringue AL, McDonald JR, Caplan L, Eisen SA, Ranganathan P. Risk of non-melanoma skin cancer in a national cohort of veterans with rheumatoid arthritis. Rheumatology (Oxford). 2011;50(8):1431-9. 
34. Chakravarty EF, Michaud K, Wolfe F. Skin cancer, rheumatoid arthritis, and tumor necrosis factor inhibitors. J Rheumatol. 2005;32(11):2130-5.

35. Silva-Fernández L, Lunt M, Kearsley-Fleet L, Watson KD, Dixon WG, Symmons DPM, et al. The incidence of cancer in patients with rheumatoid arthritis and a prior malignancy who receive TNF inhibitors or rituximab: results from the British Society for Rheumatology Biologics RegisterRheumatoid Arthritis. Rheumatology. 2016;55(11): 2033-9.

36. Winthrop KL, Saag K, Cascino MD, Pei J, John A, Jahreis A, et al. Long-term safety of rituximab in rheumatoid arthritis: analysis from the SUNSTONE registry. Arthritis Care Res (Hoboken). 2018. [Epub ahead of print].
37. Singh JA, Furst DE, Bharat A, Curtis JR, Kavanaugh AF, Kremer JM, et al. 2012 update of the 2008 American College of Rheumatology recommendations for the use of disease-modifying antirheumatic drugs and biologic agents in the treatment of rheumatoid arthritis. Arthritis Care Res. 2012;64(5): 625-39.

38. Smolen JS, Landewe R, Bijlsma J, Burmester G, Chatzidionysiou $\mathrm{K}$, Dougados $\mathrm{M}$, et al. EULAR recommendations for the management of rheumatoid arthritis with synthetic and biological diseasemodifying antirheumatic drugs: 2016 update. Ann Rheum Dis. 2017;76(6):960-77. 